\title{
Commissioning of the ATLAS High Level Trigger with Proton Collisions at the LHC
}

\author{
Brian Aagaard Petersen on behalf of the ATLAS Collaboration
}

\begin{abstract}
ATLAS is one of two general-purpose detectors at the Large Hadron Collider (LHC). The ATLAS trigger system uses fast reconstruction algorithms to efficiently reject a large rate of background events and still select potentially interesting signal events with good efficiency. After a first processing level (Level 1) using custom electronics, the trigger selection is made by software running on two processor farms, containing a total of around two thousand multi-core machines. This system is known as the High Level Trigger (HLT). To reduce the network data traffic and the processing time to manageable levels, the HLT uses seeded, step-wise reconstruction, aiming at the earliest possible rejection of background events.

The recent LHC run at the end of 2009 provided the first proton collisions at the LHC, which soon became the world's highest energy particle collider. The ATLAS trigger was essential during this period to select events with beam activity, and achieve the first ATLAS physics results. During most of the run, event selection was done by the Level 1 trigger only, with the selected data being used to commission the HLT. At the end of the 2009 run, the HLT was used to reject background events online, to cope with the increasing LHC luminosity. During the ongoing 2010 LHC run, the HLT will play a crucial role in selecting collision data for the extraction of physics results. The experience gained during the 2009 run will be essential for the coming year.

After giving an overview of the ATLAS trigger design and its innovative features, this paper focuses on the valuable experience gained in running the trigger in the the 2009 LHC run. In addition to the monitoring and configuration infrastructures, it will emphasize the trigger operation and the performance of HLT algorithms, including comparisons to the precise offline reconstruction. Finally, it will discuss the HLT plans for the 2010 run.
\end{abstract}

\section{INTRODUCTION}

$\mathbf{T}$ HE LHC [1] is a high-energy, high-intensity hadron collider built to study the Standard Model (SM) and search for physics beyond the SM. ATLAS [2] is one of two generalpurpose experiments located at the LHC. At design luminosity, $\mathcal{L}=10^{34} \mathrm{~cm}^{-2} \mathrm{~s}^{-1}$, and collision energy, $\sqrt{s}=14 \mathrm{TeV}$, the LHC will deliver approximately $10^{9}$ collisions/s to ATLAS. A multi-level trigger system has been designed for ATLAS to select only the most interesting events for later physics analysis.

The LHC commissioning with beams started with collisions at $\sqrt{s}=900 \mathrm{GeV}$ in November 2009 and collisions at $\sqrt{s}=$ $7 \mathrm{TeV}$ in March 2010. The luminosity delivered is now quickly ramping up. The ATLAS experiment is using its first data to commission the whole experiment and produce the first physics results at the energy frontier. The trigger system has

B. A. Petersen is with CERN (European Organization for Nuclear Research), Geneva, Switzerland been setup to facilitate this and at the same time prepare for a more stringent selection as the luminosity grows.

\section{ATLAS TRIGGER SyStem}

The LHC has a bunch crossing rate of $40 \mathrm{MHz}$ and an average of 25 interactions per crossing at design luminosity. The output bandwidth of the ATLAS data-acquisition system (DAQ) is limited to about $200 \mathrm{~Hz}$ due to computing resource limitations for offline event processing, storage and analysis. The rate reduction is achieved using a three level trigger system [3] illustrated in Fig. 1. At each level the selection complexity and detector granularity used increases for improved resolution and rejection power at the cost of longer decision time and thus lower processing rate.

The first level trigger (L1) is implemented in a large set of custom electronics boards that process data from all of the calorimeters and from muon detectors designed to provide fast signals for the trigger. The data are sent to the L1 processing boards through dedicated data paths and processed with coarse granularity. The calorimeter based L1 trigger searches for electromagnetic and hadronic clusters indicating the presence of electrons, photon, taus or jets. It also sums the energy from all calorimeter channels, allowing it to identify events with large total energy deposition or significant missing transverse energy. The L1 muon trigger selects muon candidate tracks based on hit coincidences across multiple layers of muon chambers. The results of the calorimeter and muon triggers are combined in the central trigger processor, which accepts events with one or more high- $p_{T}$ signals or multiple low- $p_{T}$ objects. For commissioning purposes, L1 can also accept events on one or more signals from minimum bias scintillator chambers sensitive to almost all collisions events. In case of a L1 accept, the location in $(\eta, \phi)$, known as the Region-of-Interest (RoI), of the calorimeter cluster or muon track is sent to the L2 trigger. The maximum latency of $2.5 \mu \mathrm{s}$ for the L1 trigger is set by the length of the front-end pipeline memories storing the detector signal during processing. The maximum output rate from $\mathrm{L} 1$ is $75 \mathrm{kHz}$ with the possibility to be upgraded later to $100 \mathrm{kHz}$.

The event selection in both the second level trigger (L2) and the third level trigger, known as the Event Filter (EF), is done using software algorithms running on large farms of commercial computers. The L2 and EF together are denoted as the Higher Level Trigger (HLT). After a L1 accept, the event data are read out to subdetector specific readout buffers while waiting for the L2 decision. The L2 uses fast, dedicated algorithms to confirm or reject the $\mathrm{L} 1$ decision by repeating 


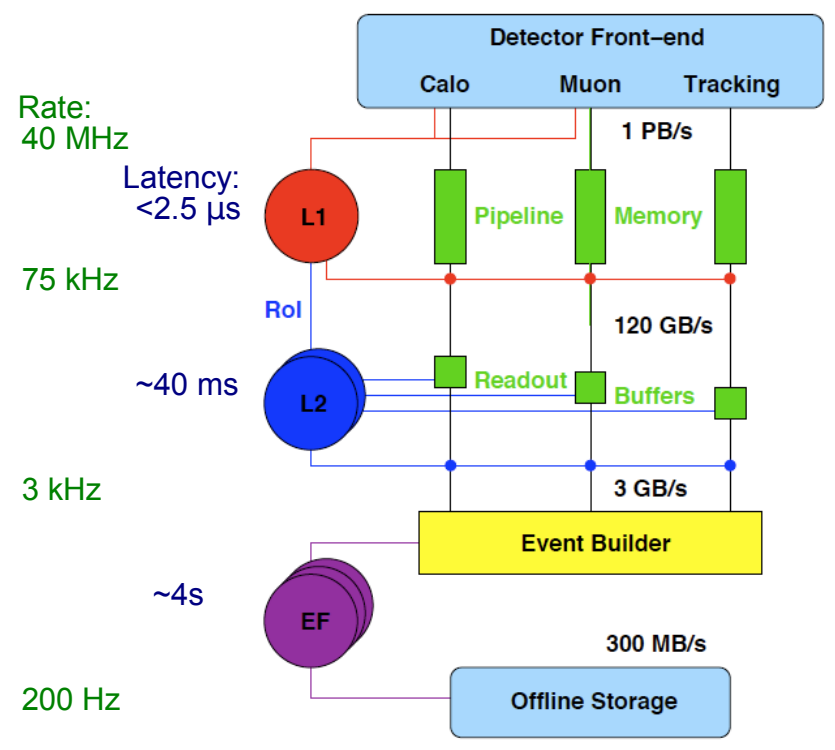

Fig. 1. Schematic overview of the three-level trigger-DAQ system in ATLAS. The average (maximum for L1) latency and accept rate has been indicated for each level together with the expected data bandwidth.

the calorimeter and/or muon reconstruction with full detector granularity. For the appropriate signatures, such as electrons or muons, L1 confirmation is combined with track reconstruction in the silicon and transition radiation trackers located inside the solenoidal field. The detector readout and reconstruction in L2 is limited to a predetermined volume around the RoI found by L1. This restricts the amount of data to be transferred and processed in $\mathrm{L} 2$ to $2-4 \%$ of the total. The final size of the L2 processing farm will be about 500 multi-core processors, setting the average processing time to about $40 \mathrm{~ms}$. The maximum $\mathrm{L} 2$ output rate is about $3 \mathrm{kHz}$, limited by the event building bandwidth.

After a L2 accept, the full event information is collected and sent to a processor in the $\mathrm{EF}$ farm. The EF therefore has access to the full event and the algorithms running are mostly using the same software as the regular offline event reconstruction, taking advantage of the full detector granularity and conditions. To have faster processing, the EF in many cases limits the reconstruction to RoIs found at L1 or refined by L2. It also uses slightly different settings than offline, such as a harder minimum $p_{T}$ cut for the track reconstruction. The final size of the EF processing farm will be about 1800 multi-core processors, setting the average processing time to a maximum of about $4 \mathrm{~s}$. The maximum HLT output rate is about $200 \mathrm{~Hz}$.

The complete trigger selection is defined by the so-called trigger menu chosen when the DAQ is configured before each run. A menu consists of many trigger chains (typically several hundred), where each chain defines the L1 and HLT selection for a single physics signature, such as an electron with $p_{T}>20 \mathrm{GeV}$ or two muons with an invariant mass around $3.1 \mathrm{GeV}$. In the HLT, a chain consists of multiple steps running one or more reconstruction and selection algorithms at each step. In case multiple chains need to run the same algorithm on the same RoI, the result from the first execution

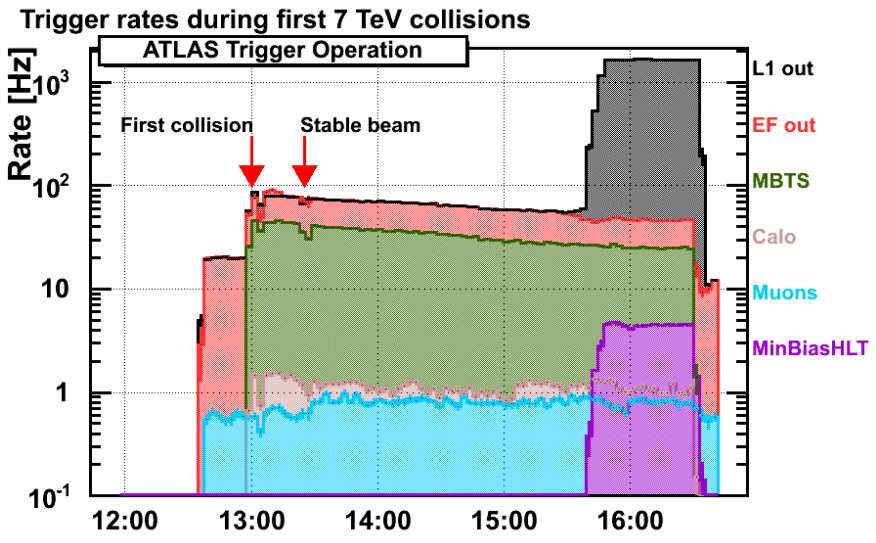

Fig. 2. Trigger rates during the first $\sqrt{s}=7 \mathrm{TeV}$ collision run (MBTS is the minimum bias scintillator based trigger). The increase in rate at around 15:40 is the enabling of an HLT based collision trigger on random triggered events.

is automatically cached in order to minimize the data processing time. A crucial feature for trigger commissioning is the ability to enable, prescale and fully disable individual chains seamlessly while taking data [4]. This for instance gives the option to very quickly disable faulty chains or to enable new chains for testing without any impact on data-taking efficiency.

\section{Commissioning of the Higher Level Trigger}

The commissioning of the HLT using the online system started more than two years ago. Simulated collisions were inserted into the readout system and processed by the HLT giving the first experience of running on a large parallel system. The commissioning period of LHC with beam in 2008 was too short to allow any testing with HLT algorithms, but both before and after the HLT was used extensively for triggering on cosmic rays [5]. The cosmic rays were used to exercise the basic muon, inner detector tracking and calorimeter clustering algorithms in the HLT. The HLT was also used to actively select a large sample of cosmic ray events with tracks crossing the tracking detectors to be used for detector commissioning [6].

For the LHC single beam and collision running in 2009 and 2010, the HLT commissioning has proceeded in several steps. During the very first beams and $\sqrt{s}=900 \mathrm{GeV}$ collisions in 2009, no HLT algorithms were running online as the event rate was very low (a few $\mathrm{Hz}$ ). Instead the HLT algorithms were exercised offline on the collision events within hours of these being recorded. After a few weeks of running offline and having verified that the algorithms behave as expected, the algorithms were enabled online in flagging-only mode. In flagging-only mode, the events are always accepted, but the HLT algorithms run normally and the decision of each chain and the reconstructed HLT objects used for making the decision is recorded into the data stream for offline analysis. The flagging-only mode can be set on a chain-by-chain basis. As described below in Sec. V, one chain was quickly moved to active HLT selection in order to measure the L1 trigger efficiency. For the first collisions at $\sqrt{s}=7 \mathrm{TeV}$ in March 2010, the HLT algorithms were again disabled initially, but 


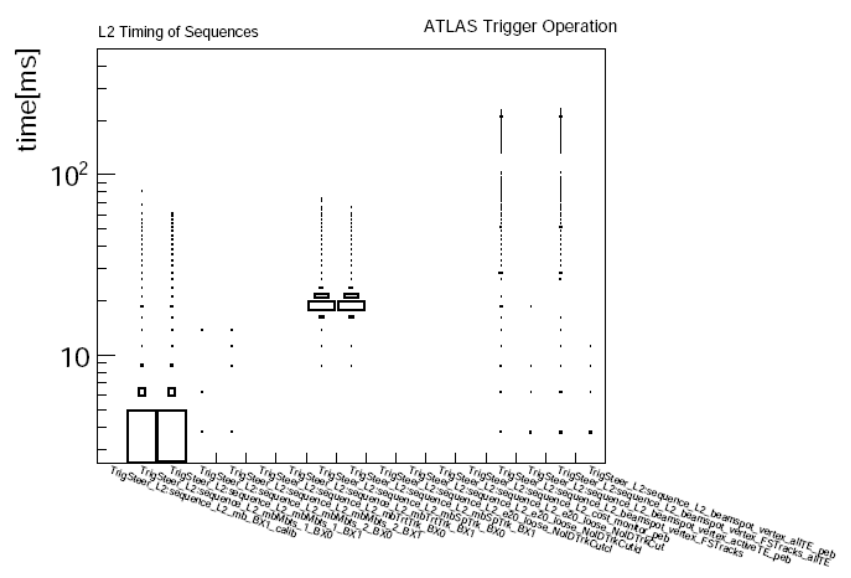

Fig. 3. Processing time versus L2 algorithms as measured online during $\sqrt{s}=900 \mathrm{GeV}$ collisions.

within two hours of data taking and fast offline processing, the HLT was turned on in flagging-only mode as shown in Fig. 2. The enabling of algorithms was done while running as described above.

During the first half year of beam operation, the LHC has been ramping up the peak luminosity from $7 \times 10^{26} \mathrm{~cm}^{-2} \mathrm{~s}^{-1}$ to $6 \times 10^{28} \mathrm{~cm}^{-2} \mathrm{~s}^{-1}$. This has been sufficiently low to allow the HLT to continue running in flagging-only mode and controlling the output rate by prescaling minimum bias collision triggers at L1. The trigger menu has however evolved several times to better adapt to the running conditions and commission a larger part of the HLT. For the $\sqrt{s}=900 \mathrm{GeV}$ running period in 2009, the HLT had about 170 chains running in flagging-only mode, while for the first $\sqrt{s}=7 \mathrm{TeV}$ collisions this was increased to about 220 chains. The final commissioning menu currently in use has about 420 HLT chains. Many of the chains are only included to ensure all of the HLT algorithms are exercised even at very low luminosity. This menu is expected to be used until the luminosity reaches about $10^{30} \mathrm{~cm}^{-2} \mathrm{~s}^{-1}$. It will be used for the first active HLT selection as some of the chains with the lowest $p_{T}$ thresholds will need to go into active mode when the luminosity reaches about $10^{29} \mathrm{~cm}^{-2} \mathrm{~s}^{-1}$. The final physics menu, designed for luminosities of $10^{30} \mathrm{~cm}^{-2} \mathrm{~s}^{-1}$ to $10^{32} \mathrm{~cm}^{-2} \mathrm{~s}^{-1}$ is expected to have about 300 chains and to be deployed at the end of June 2010.

Running the HLT online in flagging-only mode is very useful for commissioning the HLT quickly. More than 150 million events have now been processed by the HLT and thanks in part to the extensive testing with simulated and cosmic ray data beforehand, no algorithm related crashes has been observed online. Typically a dozen events per day cause processing timeouts, but these have all been found to be due to massive cosmic showers with very high occupancy in the muon detectors leading to very long processing times in commissioning chains for muons. This is an acceptable rate during commissioning, particularly as it also helps commission the offline handling of timeout and other HLT failure events. The monitoring of the HLT online has also been exercised

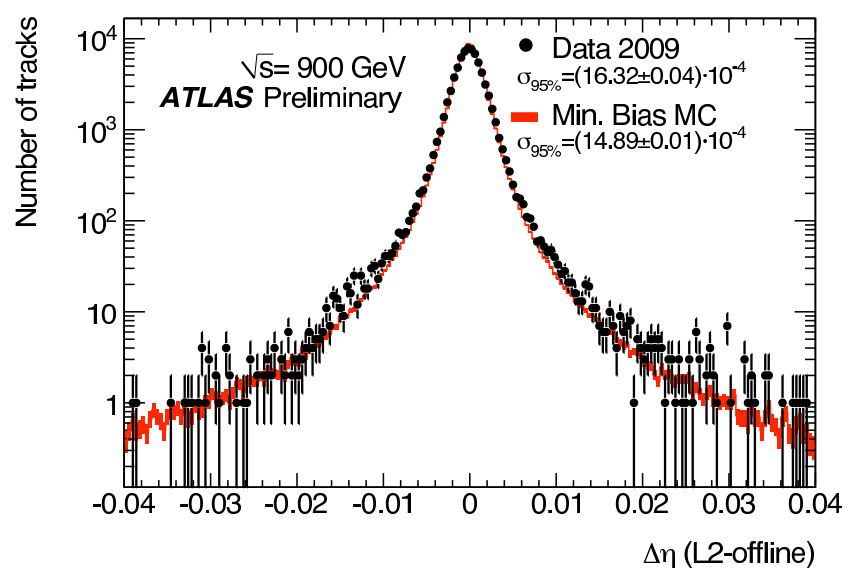

Fig. 4. Difference in the $\eta$ track parameter between the L2 tracks and their corresponding matched offline tracks.

thoroughly by having the HLT running. Many of the software changes deployed during the first six months have indeed been monitoring improvements allowing shifters and experts to more easily check that the HLT algorithms are running properly. Finally the performance of the HLT algorithms is measured. The rejection factor of each HLT chain is measured online as well as the processing time for each algorithm as shown in Fig. 3. Having the HLT decision recorded, but not used, allows the efficiency of each chain with respect to offline reconstruction to be measured before a chain is put into active mode as explained in the next section.

\section{HLT PERFormance StUdies}

For each chain almost all information used to make the trigger decision, such as reconstructed calorimeter clusters and track parameters, are recorded into the raw data stream along with the trigger decision. This information is propagated to the reconstructed event data during the offline event reconstruction. It is therefore possible to directly compare the online reconstructed objects with the equivalent offline ones and use this to understand any observed trigger inefficiencies. The most accurate efficiencies are obtained when measured on the signal events of interest, such as offline reconstructed high- $p_{T}$ muons from $W \rightarrow \mu \nu_{\mu}$ decays. The statistics for most signal processes are however still very low and one therefore has to use for instance muons from $B$ decays or even $K$ and $\pi$ decays in flight, for which the trigger is not as efficient.

\section{A. HLT Tracking}

Most signatures in the trigger are using tracks as part of the selection. Besides understanding the track reconstruction efficiency, it is also important to look at the track parameter resolution as those are used for instance for $E / p$ selections for electrons or impact parameter selections for $B$-physics triggers. Normally HLT tracks are reconstructed only in the RoI for a given trigger chain, however at the initial low luminosity and particularly at $\sqrt{s}=900 \mathrm{GeV}$ there are few RoIs accepted by the L 2 calorimeter or muon algorithms. The commissioning menus therefore have special chains running 


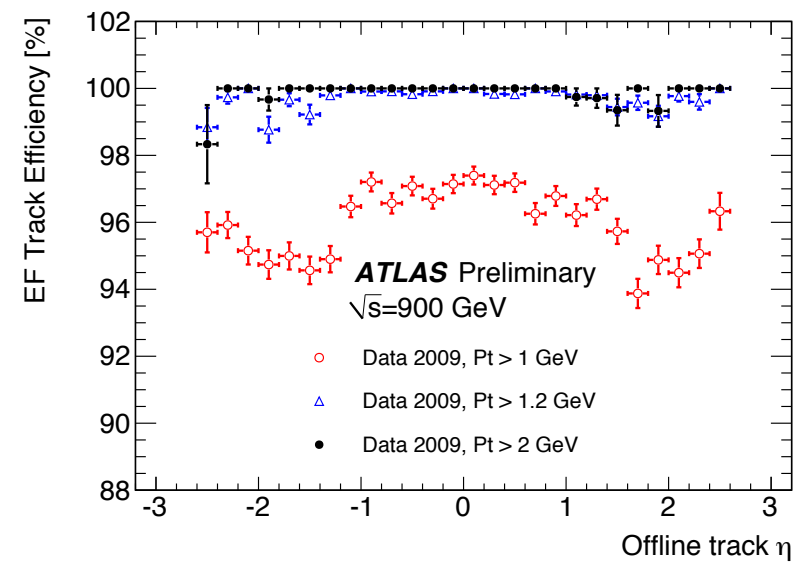

Fig. 5. Efficiency for EF tracking with respect to offline as a function of the offline track rapidity, $\eta$. Efficiencies are shown for three different minimum offline track $p_{T}$ values.

both the L2 and EF tracking algorithms on almost every collision event in a special full-scan mode. In this mode the tracking tries to reconstruct all tracks with $p_{T}>1 \mathrm{GeV}$ in the full inner detector.

The reconstructed HLT tracks are compared [7] to both offline reconstructed tracks and to HLT tracks reconstructed in simulated minimum bias events. The offline selected tracks are required to be well-reconstructed with a minimum number of silicon detector hits and impact parameters close to the interaction point. Offline and HLT tracks are matched by requiring $\Delta R=\sqrt{\Delta \eta^{2}+\Delta \phi^{2}}<0.1$ though the exact value of this matching is found to have very little impact on the measured efficiencies. Comparing the number of pixel and SCT hits on the L2 tracks between data and simulation, very good agreement is found and the missing hits with respect to the hits used on offline tracks also agrees between data and simulation. For EF tracks, the number of hits is compared to the offline tracks directly and again good agreement is found. The track parameter resolution measured with respect to offline is as expected. An example comparison is shown in Fig. 4. The resolution for EF tracks is a factor 3-5 better than the L2. The tracking efficiency of both the L2 and EF is found to approach $100 \%$ quickly as the track $p_{T}$ increases above $1 \mathrm{GeV}$ as expected. This can be seen in Fig. 5 for EF tracks. been measured with the RoI-based

The full-scan L2 tracking algorithm described above can run at a rate of up to several $\mathrm{kHz}$ without taking too many resources from the trigger system. The full-scan tracks are used to fit the primary vertex position on an event-by-event basis. The vertex results from several minutes of data taking are then combined into a precise estimate of the luminous region [8]. The position and size are fed back to LHC operations to help understand and optimize the machine performance. The location of the interaction region is also needed by trigger algorithms that look for detached vertexes, such as $b$ jet tagging algorithms. It is therefore foreseen to feed back the interaction region location and size to the HLT processes on a regular basis during a run [4].

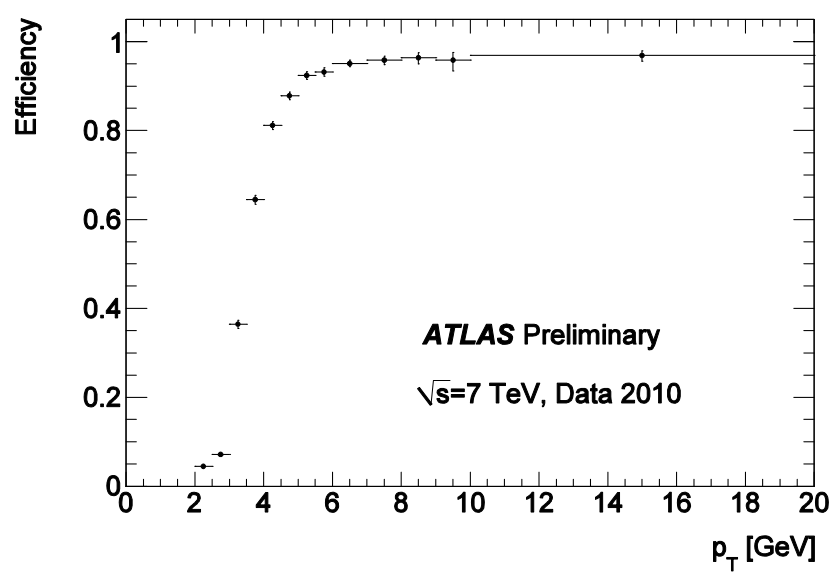

Fig. 6. Efficiency of L2 muon standalone algorithm relative to offline reconstructed muons with a nominal threshold set to $4 \mathrm{GeV}$ as a function of the transverse momentum $p_{T}$ measured by the offline reconstruction.

\section{B. Calorimeter-based HLT Triggers}

The calorimeters together with the muon detectors are the starting point for all high- $p_{T}$ triggers in ATLAS. The performance of both the basic calorimeter clustering algorithms and the combined reconstruction of for instance electron, photon and jet objects has been studied in detail [9]. The distributions of the variables used for selecting electrons, etc. are generally found to be in good agreement with simulations. Therefore signal efficiencies as measured with respect to offline reconstructed objects are observed to be roughly the same in simulations as well.

\section{HLT Muon Triggers}

For muon triggers the performance of the complete HLT muon reconstruction has been studied in the $\sqrt{s}=900 \mathrm{GeV}$ and $\sqrt{s}=7 \mathrm{TeV}$ data samples [10], though with very limited statistics in the former.

At L2, a fast pattern recognition algorithm is used to find muon tracks using additional muon detector hits in the RoIs found by the L1 muon trigger. The track parameters are then calculated using lookup tables and a minimum $p_{T}$ requirement is applied. If the standalone reconstruction passes the event, inner detector tracks are reconstructed in the RoI. If found, an average transverse momentum is calculated for improved resolution and event rejection. To study the algorithm performance, offline muon candidates reconstructed in both muon chamber and inner detector are used in events where the L1 muon trigger has fired with an RoI that match the offline muon. This ensures the existence of the seed for L2. The standalone L2 muon reconstruction for these muon candidate is found to be above $95 \%$. The lookup table for calculating momentum has not been optimized yet, leading to a slightly slower than expected turn-on curve as shown in Fig. 6. The efficiency for combining with an inner detector track is almost $100 \%$ for tracks in the barrel, $|\eta|<1.05$, while in the endcap there are some inefficient regions, mainly due to nonoptimal extrapolation of the RoI from the muon detector to the tracking detectors. The inefficiencies are being solved as 


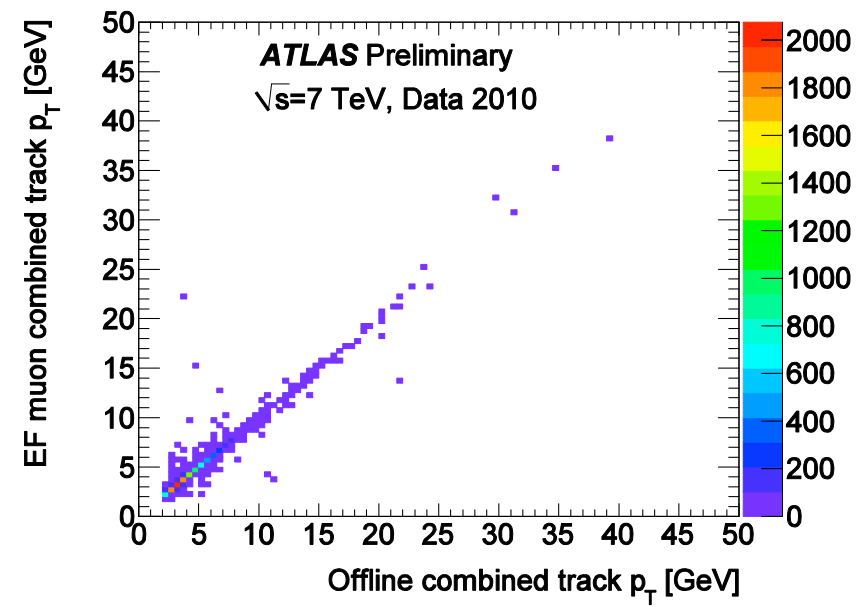

Fig. 7. Comparison of the transverse momentum measured by the EF muon combined algorithm and the offline combined muon reconstruction.

part of the commissioning work, though a larger sample of well-reconstructed muons are needed to reach the ultimate performance.

The EF muon reconstruction is based on offline algorithms for standalone muon and inner detector reconstruction and a combined track fit of the standalone objects. For performance studies, the same offline reconstructed muon candidates as in the L2 study are used. To disentangle L2 and EF performance, the L2 is not required to reconstruct the muon candidate. With this selection, very good agreement between EF and offline track parameters are seen as illustrated in Fig. 7 for the transverse momentum. The EF muon trigger efficiency is above $98 \%$ when $p_{T}>4 \mathrm{GeV}$.

\section{Active HLT Trigger Selection}

Until the end of May 2010, only one HLT chain has been used to actively select events for physics analysis. The first published ATLAS physics analysis is the measurement of charged particle multiplicity at $\sqrt{s}=900 \mathrm{GeV}$ [11]. The event selection for this analysis uses collision events selected by the L1 minimum bias scintillator trigger. It is therefore important to measure and correct for the efficiency of this trigger. Ideally pure randomly selected events should be used for this measurement. However, at low luminosity the bandwidth required to obtain sufficient statistics is too high. Instead the L1 was setup to randomly select events at a rate of $1-5 \mathrm{kHz}$ and a simple HLT chain was used to select collision events with minimal bias. The chain required that the number of hits in the pixel and SCT detectors be a few counts above the noise level and that the EF was able to reconstruct at least one track with $p_{T}>500 \mathrm{MeV}$. Fig. 8 shows the measured efficiency for using three different hit multiplicities in the L1 minimum bias scintillator trigger as a function of the offline reconstructed track multiplicity. Based on this, it was possible to choose the most efficient and unbiased L1 trigger for the analysis and to apply a small correction for the remaining inefficiency.

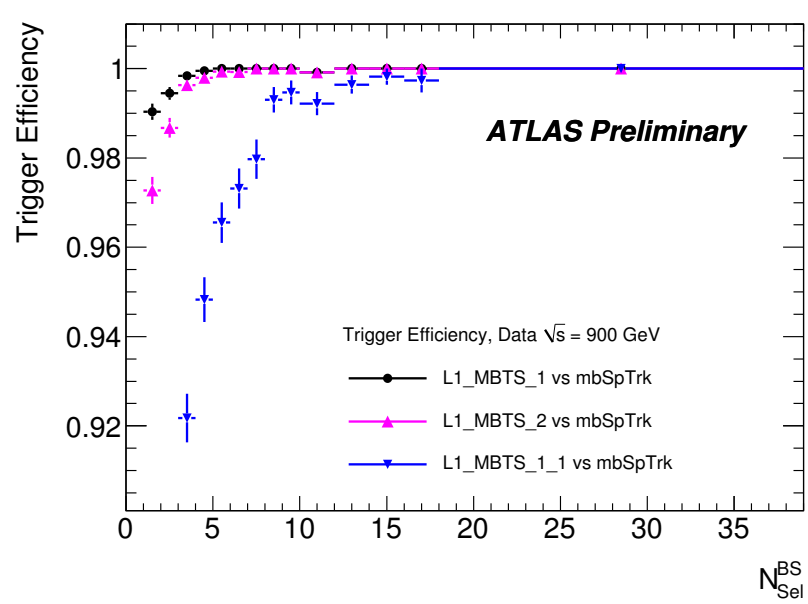

Fig. 8. The efficiencies of three different minimum bias L1 collision triggers with respect to HLT+Offline selected collisions as a function of the number of offline selected tracks.

\section{Summary AND OUtLOOK}

The ATLAS experiment has developed a flexible high-level trigger system for triggering on a diverse set of physics signals. The system is currently being commissioned with the first LHC collision data. All basic selection algorithms are routinely executed fully online, though only one is being used to actively select events. No significant operational problems with the HLT have been found. The trigger performance in terms of efficiency and trigger object resolution with respect to offline reconstructed signals is being evaluated. The performance is mostly found to be consistent with expectations from simulated data.

The luminosity delivered from LHC is rapidly increasing and the HLT will therefore become a major component in the event selection shortly. This will happen in steps, enabling the lowest $p_{T}$ thresholds first as needed by the increasing L1 trigger rates. The commissioning of high- $p_{T}$ object triggers and more complex triggers will proceed in parallel. Over the next months the LHC luminosity is expected to rise by more than two orders of magnitude and by the end of it, most of the HLT will be in active selection mode.

\section{REFERENCES}

[1] L. Evans and P. Bryant, LHC Machine, Journal of Instrumentation, vol. 3, p. S08001,2008.

[2] G. Aad et al., The ATLAS Experiment at the CERN Large Hadron Collider, Journal of Instrumentation, vol. 3, p. S08003, 2008.

[3] G. Aad et al., Expected Performance of the ATLAS Experiment Detector, Trigger and Physics, CERN-OPEN-2008-020, 2009.

[4] F. Winklmeier, Real-time configuration changes of the ATLAS High Level Trigger, in these proceedings, 2010.

[5] I. Aracena, Operational Experience of the ATLAS High Level Trigger with Single-Beam and Cosmic Rays, proceedings of 2009 IEEE Nuclear Science Symposium and Medical Imaging Conference, 2009.

[6] G. Aad et al., The ATLAS Inner Detector commissioning and calibration, accepted by European Physics Journal C, 2010.

[7] E. Christidi, Performance of the ATLAS Inner Detector Trigger algorithms in p-p collisions at centre-of-mass energy of $900 \mathrm{GeV}$, in these proceedings, 2010.

[8] R. Bartoldus, D. W. Miller, D. Su, and F. Winklmeier, Online Measurement of LHC Beam Parameters with the ATLAS High Level Trigger, in these proceedings, 2010. 
[9] M. Wielers, ATLAS e/g/tau/jet/etmiss High Level Trigger Algorithms Performance with first LHC collisions, in these proceedings, 2010.

[10] M. Owen, Commissioning of the ATLAS Muon High Level Trigger with cosmics and beam collisions at the LHC, in these proceedings, 2010.
[11] G. Aad et al., Charged-particle multiplicities in pp interactions at $\sqrt{s}=$ $900 \mathrm{GeV}$ measured with the ATLAS detector at the LHC, Phys. Lett. B 688, 1, p. 21-42, 2010. 